\title{
Pyrrolizidine Alkaloid Profiles of Some Senecio Species from Egypt
}

\author{
Assem El-Shazly \\ Department of Pharmacognosy, Faculty of Pharmacy, Zagazig University, Egypt \\ Z. Naturforsch. 57c, 429-433 (2002); received January 4/February 1, 2002 \\ Pyrrolizidine Alkaloids, Senecio aegyptius, Senecionine
}

Alkaloid profiles of two Egyptian Senecio species (Senecio aegyptius var. discoideus and $S$. desfontainei) in addition to a cultivated species ( $S$. cineraria) were studied using capillary GLC and GLC-mass spectrometry with respect to pyrrolizidine alkaloids (PAs). Four alkaloids were identified in S. aegyptius var. discoideus, 8 in $S$. desfontainei and 13 in S. cineraria. Some of these alkaloids have not been reported from these plants. The alkaloidal pattern of different plant organs (flowers, leaves, stem, root) were also investigated. Senecionine has been found to be a one of the major alkaloid in all studied species, it was isolated and its structure was elucidated by ${ }^{1} \mathrm{H}$ - and ${ }^{13} \mathrm{C}-\mathrm{NMR}$.

\section{Introduction}

Numerous taxa of the genus Senecio (Compositae) are rich in PAs exhibiting hepatotoxic, mutagenic, carcinogenic and antitumor activities (reviews in Mattocks 1986; Rizk 1990; Roeder 1995, 1999; Hartmann and Witte 1995; Roberts and Wink 1998).

Reviewing the current literature 3 PAs were previously identified in S. aegyptius (Gharbo and Habib 1969; Habib 1981), 4 in S. desfontainei (Gharbo and Habib 1969; Habib 1981) and 5 in $S$. cineraria (Habib 1974; Rizk 1990); no information could be obtained concerning the alkaloid content of $S$. aegyptius var. discoideus. In the present work and in the course of our study on the Egyptian plants containing PAs (El-Shazly et al. 1996a,b; 1998; 1999) we have investigated the alkaloidal pattern of flowers, leaves, stem and root of wild $S$. aegyptius var. discoideus, $S$. desfontainei and the cultivated $S$. cineraria species by sensitive phytochemical methods (GLC and GLC-MS). Since capillary GLC in combination with mass spectrometry is a rapid and powerful technique for the analysis of complex PA mixtures (Bicchi et al., 1991; Witte et al., 1993; Stelljes et al., 1991; ElShazly et al., 1996a-d; 1998; 1999), we were able to unambiguously identify most PAs in these plants and have found a number of alkaloids which were reported for the first time in the studied species.

\section{Materials and Methods}

\section{Plant material}

Flowering plant of S. aegyptius var. discoideus Boiss. was collected from the Nile banks at the vicinity of Benha in April 2000. S. desfontainei Druce (= S. coronopifolius Desf.) was collected from sandy area near Bilbeis in March 2000. S. cineraria Dc. (syn. Cineraria maritima L.) was collected from plants cultivated in the experimental station of the Faculty of Pharmacy, Zagazig University in May 2000. Identification of these plants was confirmed by Dr. H. Abdel Baset, Faculty of Science, Zagazig University. Voucher specimens are deposited in the Herbarium of the Department of Pharmacognosy, Faculty of Pharmacy, Zagazig University. The different plant organs were separated, air dried and powdered.

\section{Alkaloid extraction}

Air dried plant materials ( $10 \mathrm{~g}$ each) were separately extracted twice with $0.5 \mathrm{~N}$ HCI $(500 \mathrm{ml})$ through homogenization by Ultra-turrax and left to stand for one hour. The resulting extracts were combined, then defatted with $\mathrm{CH}_{2} \mathrm{CI}_{2}$. Half of the aqueous phase was made alkaline with $25 \%$ $\mathrm{NH}_{4} \mathrm{OH}$ solution ( $\left.\mathrm{pH} 10\right)$ and extracted with $\mathrm{CH}_{2} \mathrm{CI}_{2}$. The resulting extract was dried over anhydrous $\mathrm{Na}_{2} \mathrm{SO}_{4}$ and evaporated to dryness to afford the free tertiary bases. To investigate the presence of PA $N$-oxides, the second aliquot of the aqueous phase was brought to $2 \mathrm{~N} \mathrm{HCI}$ and reduced with $\mathrm{Zn}$ dust under continuous stirring 
overnight, filtered and treated as above to obtain the total alkaloids (tertiary bases and $N$-oxides) in the form of tertiary PA bases. The dried residues were weighed and kept at $4{ }^{\circ} \mathrm{C}$ for GLC and GLCMS analysis. PLC [silica gel $\mathrm{F}_{254} \mathrm{CHCI}_{3}-\mathrm{MeOH}-$ $\left.\left.\mathrm{NH}_{4} \mathrm{OH}(25 \%), 85: 15: 1\right)\right]$ of the crude PA tertiary bases of of $S$. aegyptius var. discoideus resulted in the isolation of a major alkaloid (4) with $\mathrm{R} f 0.68$.

\section{Capillary GLC analysis}

A Carlo Erba ICU 600 gas chromatograph equipped with FID, Spectra Physics integrator and DB1 fused silica capillary column (15 $\mathrm{m} \times$ $0.317 \mathrm{~mm}$ i.d. $0.25 \mu \mathrm{m}$ film thickness) was employed. GLC condition: carrier gas $\mathrm{He}(2 \mathrm{ml} /$ min); detector temp. $300{ }^{\circ} \mathrm{C}$; injector temp. $250{ }^{\circ} \mathrm{C}$; oven temp. program, initial temp. $150{ }^{\circ} \mathrm{C} 5 \mathrm{~min}$ isothermal, $150-300{ }^{\circ} \mathrm{C} 10{ }^{\circ} \mathrm{C} / \mathrm{min} .300{ }^{\circ} \mathrm{C}, 15 \mathrm{~min}$ isothermal. Retention index (RI): Kovats indices (Kovats, 1958) were calculated with respect to a set of co-injected even number hydrocarbons (C10 - C28). Each RI was subjected to library search by comparison with references RI and MS.

\section{GLC-MS analysis}

A Carlo Erba HRGC 4160 gas chromatograph equipped with OV1 capillary column $(30 \mathrm{~m}$, $0.25 \mathrm{~mm}$ i.d., $0.25 \mu \mathrm{m}$ film thickness) coupled to a quadrupole mass spectrometer Finnigan MAT 4500 was employed for PA analysis. EI-mass spectra were recorded at $45 \mathrm{eV}$. Condition: injector $250{ }^{\circ} \mathrm{C}$; temp. program $120^{\circ} \mathrm{C} 3 \mathrm{~min}$ isothermal, $120-300{ }^{\circ} \mathrm{C} 6{ }^{\circ} \mathrm{C} / \mathrm{min} ., 300{ }^{\circ} \mathrm{C} 10 \mathrm{~min}$ isothermal; split ratio 1: 20; carrier gas $\mathrm{He} 50 \mathrm{kPa}$.

\section{NMR measurements}

${ }^{1} \mathrm{H}$ and ${ }^{13} \mathrm{C}$ NMR spectra were recorded on Varian instrument in $\mathrm{CDCI}_{3}$, at 300 and $75 \mathrm{MHz}$, respectively.

Table I. Pyrrolizidine alkaloids of Senecio aegyptius var. discoideus, S. desfontainei and S. cineraria separated by capillary GLC and identified by GLC-MS.

\begin{tabular}{|c|c|c|c|c|}
\hline Alkaloid* & RI & $\mathrm{M}^{+}$ & B. P. & Reference \\
\hline 1 9-Methyl-didehydroretronecine & 1387 & 167 & 136 & 1 \\
\hline 2 5,6-Dihydro-7,9-dimethoxy-7H-pyrrolizine & 1413 & 181 & 150 & 1 \\
\hline 3 Senecivernine & 2299 & 335 & 120 & 2,3 \\
\hline 4 Senecionine & 2310 & 335 & 136 & 4 \\
\hline 5 Seneciphylline & 2327 & 333 & 120 & 5 \\
\hline 6 Spartioidine & 2359 & 333 & 120 & 6 \\
\hline 7 Integerrimine & 2367 & 335 & 119 & 7 \\
\hline 8 Jacobine & 2432 & 351 & 120 & 3 \\
\hline 9 Riddelliine & 2471 & 349 & 119 & 2 , \\
\hline 10 Acetylseneciphylline & 2483 & 357 & 136 & 1 \\
\hline 11 Jacoline & 2508 & 369 & 120 & 1,3 \\
\hline 12 Retrorsine & 2539 & 351 & 120 & 7 \\
\hline 13 Jaconine & 2548 & 387 & 120 & 1,3 \\
\hline 14 Usaramine & 2607 & 351 & 119 & 6 \\
\hline 15 Otosenine & 2631 & 381 & 151 & 8 \\
\hline 16 Florosenine & 2776 & 423 & 43 & 1,9 \\
\hline 17 Doronine & 2818 & 459 & 43 & 1,9 \\
\hline 18 PA1* & 2543 & 351 & 80 & \\
\hline 19 PA2* & 2563 & 351 & 138 & \\
\hline 20 PA $3 *$ & 2595 & 351 & 138 & \\
\hline
\end{tabular}

1= Tei, 2000; 2= Borstel et al., 1989; 3= Witte et al., 1992; Witte et al., 1993; 5= Roeder and Abdel Ghani 1990; 6= Pieters and Vlientink 1988; 7= Ray et al., 1987; 8 = Resch et al., 1982; 9= Krebs et al., 1996.

$\mathrm{RI}=$ retention index, $\mathrm{M}^{+}=$molecular weight, $\mathrm{B}$. $\mathrm{P}$. = base peak.

Identified compounds are listed in order of elution from OV-1 column under condition listed in experimental section.

* Mass spectra of unknown compounds; PA1 (18), GLC-EIMS, m/z (rel. int.): $\left[\mathrm{M}^{+}\right] 351$ (10), 333 (20), 262 (8), 137 (27), 136 (27), 125 (60), 120 (70), 119 (67), 111 (60), 106 (56), 94 (30), 80 (100), 43 (85).

PA2 (19), GLC-EIMS, m/z (rel. int.): [M+] 351 (58), 282 (10), 227 (38), 138 (100), 125 (30), 94 (60), 93 (53), 80 (20), $43(43)$.

PA3 (20), GLC-EIMS, $m / z$ (rel. int.): $\left[\mathrm{M}^{+}\right] 351$ (8), 333 (4), 227 (10), 139 (30), 138 (100), 137 (40), 125 (40), 120 (13), 106 (10), 94 (65), 93 (60), 80 (30). 


\section{Results and Discussion}

Capillary GLC and GLC-mass spectrometry is a valuable method for resolving and identification of complex PA mixtures even in trace amounts or diasteromeric forms. Combination of $\mathrm{M}^{+}$, RI and mass group fragmentation pattern provided unequivocal identification of most of these alkaloids. The identification of the compounds was established by direct comparison (MS, RI) with authentic alkaloids or by comparison with literature data. As PAs often occur both as tertiary bases and the corresponding $N$-oxides, the aqueous acidic extract of the plant material was worked up to give separate estimation of the tertiary bases and of the total alkaloid content (tertiary bases and reduced $N$-oxides) as well. The alkaloid contents were estimated gravimetrically and given as $\mathrm{w} / \mathrm{w} \%$ of dried plant materials. 17 PAs were unambiguously identified in the crude tertiary bases of $S$. aegyptius var. discoideus, $S$. desfontainei and $S$. cineraria species (Table I). In all studied species most of PAs were present as $N$-oxide rather than the corresponding tertiary bases (see tertiary bases and $N$-oxides) (Table II).
Senecio cineraria is grown in Egypt as an ornamental plant. Previous studies (Habib 1974, Rizk 1990) on this species resulted in isolation and identification of 5 PAs namely; senecionine, seneciphylline, jacobine, otosenine and retrorsine. In the present study only 4 of the previously identified components and additional 9 other alkaloids namely 9-methyl-didehydroretronecine, 5,6-dihydro-7,9-dimethoxy-7H-pyrrolizine, senecivernine, integerrimine, jacoline, jaconine, usaramine, florosenine and doronine are now reported and identified for the first time as alkaloids in S. cineraria. On the other hand we have not found any trace of retrorsine. Highest concentration occurs in the flower heads, while other organs contain relatively smaller amounts (Table II).

Concerning $S$. desfontainei, eleven peaks were observed in the GLC and GLC-MS of which 8 alkaloids could be identified three of them were previously reported in this plant viz. senecionine, seneciphylline and riddelliine (Gharbo and Habib 1969; Habib 1981). New alkaloids for this plant are: senecivernine, spartioidine, integerrimine, acetylseneciphylline and retrorsine. The presence

Table II. Pyrrolizidine alkaloid profiles and percentage in Senecio aegyptius var. discoideus, S. desfontainei and S. cineraria using capillary gas chromatography.

\begin{tabular}{|c|c|c|c|c|c|c|c|c|c|c|c|c|c|c|c|c|c|c|c|c|c|c|c|c|}
\hline \multirow[t]{3}{*}{ Alkaloid* } & \multicolumn{8}{|c|}{ Senecio aegyptius var. discoideus } & \multicolumn{8}{|c|}{ S. desfontainei } & \multicolumn{8}{|c|}{ S. cineraria } \\
\hline & $\mathbf{F}$ & & 1 & $\mathbf{L}$ & & $\mathbf{S}$ & 1 & $\mathbf{R}$ & & $\mathbf{F}$ & 1 & $\mathbf{L}$ & & $\mathbf{S}$ & & $\mathbf{R}$ & & $\mathbf{F}$ & $\mathbf{I}$ & $\mathbf{L}$ & & $\mathbf{S}$ & $\mathbf{R}$ & $\mathbf{R}$ \\
\hline & Ter. & Tot. & Ter. & Tot. & Ter. & Tot. & Ter. & Tot. & Ter. & Tot. & Ter. & Tot. & Ter. & Tot. & Ter. & Tot. & Ter. & Tot. & Ter. & Tot. & Ter. & Tot. & Ter. & Tot. \\
\hline 1 & - & - & - & - & - & - & - & - & - & - & - & - & - & - & - & - & $\operatorname{tr}$ & $\operatorname{tr}$ & - & - & - & 5.8 & - & $\operatorname{tr}$ \\
\hline 2 & - & - & - & - & - & - & - & - & - & - & - & - & - & - & - & - & $\operatorname{tr}$ & $\operatorname{tr}$ & - & - & - & $\operatorname{tr}$ & - & - \\
\hline 3 & 9.2 & 10.2 & 6.9 & 21.4 & 18.1 & 26.8 & $\operatorname{tr}$ & 18.9 & 1.9 & $\operatorname{tr}$ & 4.1 & $\operatorname{tr}$ & 3.9 & $\mathrm{tr}$ & 1.8 & $\operatorname{tr}$ & - & $\operatorname{tr}$ & - & 2.9 & - & $\operatorname{tr}$ & - & 0.5 \\
\hline 4 & 52.3 & 68.1 & 12.5 & 49.1 & 24.6 & 41.5 & $\operatorname{tr}$ & 53.7 & 42.1 & 55.4 & 21.3 & 44.4 & 37.4 & 28.3 & 52.3 & 70.6 & 8.6 & 1.5 & - & 1.8 & 3.6 & 16.8 & 29.5 & 33.6 \\
\hline 5 & 23.9 & 21.7 & 28.9 & 28.8 & 32.9 & 30.6 & 55.4 & 27.5 & 20.3 & 34.6 & 3.5 & 27.7 & 4.5 & 17.9 & 3.6 & 8.4 & - & 0.4 & - & $\mathrm{tr}$ & $\operatorname{tr}$ & $\operatorname{tr}$ & 9.2 & 11.4 \\
\hline 6 & 14.6 & $\operatorname{tr}$ & 51.8 & 0.7 & 24.4 & 1.1 & 44.6 & $\operatorname{tr}$ & $\operatorname{tr}$ & $\operatorname{tr}$ & 8.5 & $\mathrm{tr}$ & 5.1 & $\operatorname{tr}$ & 9.5 & 1.1 & - & - & - & - & - & - & - & - \\
\hline 7 & - & - & - & - & - & - & - & - & 9.1 & 8.0 & 13.8 & 11.0 & 14.6 & 12.3 & 5.8 & 11.1 & 0.8 & 0.8 & 7.3 & $\operatorname{tr}$ & 7.4 & 5.9 & 1.3 & 6.9 \\
\hline 8 & - & - & - & - & - & - & - & - & - & - & - & - & - & - & - & - & 8.6 & 10.9 & 28.1 & 13.8 & 16.4 & 14.7 & 9.3 & 31.1 \\
\hline 9 & - & - & - & - & - & - & - & - & $\operatorname{tr}$ & $\operatorname{tr}$ & $\operatorname{tr}$ & $\operatorname{tr}$ & $\operatorname{tr}$ & $\operatorname{tr}$ & $\operatorname{tr}$ & $\operatorname{tr}$ & - & - & - & - & - & - & - & - \\
\hline 10 & - & - & - & - & - & - & - & - & $\operatorname{tr}$ & $\operatorname{tr}$ & - & $\mathrm{tr}$ & - & $\operatorname{tr}$ & - & $\operatorname{tr}$ & - & - & - & - & - & - & - & - \\
\hline 11 & - & - & - & - & - & - & - & - & - & - & - & - & - & - & - & - & 2.4 & 3.0 & 10.3 & 7.8 & 9.8 & 5.8 & 4.1 & 6.2 \\
\hline 12 & - & - & - & - & - & - & - & - & - & - & - & - & - & - & - & - & - & - & - & - & - & - & - & - \\
\hline 13 & - & - & - & - & - & - & - & - & - & - & - & - & - & - & - & - & 3.8 & 9.7 & 10.5 & 63.9 & 13.2 & 39.7 & 1.7 & 3.7 \\
\hline 14 & - & - & - & - & - & - & - & - & - & - & - & - & - & - & - & - & 0.2 & 0.9 & 25.5 & $\operatorname{tr}$ & 10.7 & $\operatorname{tr}$ & 0.7 & 3.4 \\
\hline 15 & - & - & - & - & - & - & - & - & - & - & - & - & - & - & - & - & 20.1 & 33.3 & $\operatorname{tr}$ & 2.9 & 23.9 & 7.4 & 4.2 & $\operatorname{Tr}$ \\
\hline 16 & - & - & - & - & - & - & - & - & - & - & - & - & - & - & - & - & 42.1 & 37.5 & $\operatorname{tr}$ & 2.9 & 13.6 & $\operatorname{tr}$ & 1.3 & $\operatorname{tr}$ \\
\hline 17 & - & - & - & - & - & - & - & - & - & - & - & - & - & - & - & - & 3.4 & 10.9 & $\operatorname{tr}$ & 3.9 & 30.8 & $\operatorname{tr}$ & $\operatorname{tr}$ & $\operatorname{tr}$ \\
\hline $\begin{array}{l}\text { Total } \\
\text { alkaloid** }\end{array}$ & 0.18 & 0.47 & 0.13 & 0.26 & 0.14 & 0.18 & 0.1 & 0.1 & 0.21 & 0.61 & 0.06 & 0.13 & 0.06 & 0.11 & 0.12 & 0.10 & 0.26 & 0.23 & 0.05 & 0.12 & 0.05 & 0.21 & 0.04 & 0.12 \\
\hline
\end{tabular}

$*$ = Numbers of compounds as in Table $\mathbf{I}, * *=\mathrm{mg} / 100 \mathrm{mg} \mathrm{w} / \mathrm{w}$ dried plant material.

$\mathrm{F}=$ flowers, $\mathrm{L}=$ leaves, $\mathrm{S}=$ stem, $\mathrm{R}=$ root; $\operatorname{tr}=$ trace amounts $(<0.1) ;-=$ not detected.

Ter. $=$ tertiary bases (before $Z n$ reduction), Tot. = total alkaloids (tertiary bases and N-oxides after $Z n$ reduction). 
of otosenine previously reported from this plant was not confirmed. Three unidentified PAs: PA1 (RI 2543), PA2 (RI 2563) and PA3 (RI 2595) were detected in delayed elution components in GLC and GLC-MS (Table I) and their individual percentages were only in small or trace amounts. The mass spectrum of these alkaloids exhibited a molecular ion $\left[\mathrm{M}^{+}\right]$at $m / z$ 351. This $\left[\mathbf{M}^{+}\right]$indicates a senecivernine, senecionine or integerrimine containing additional oxygen.

Three PAs namely senecionine, otosenine and riddelliine have been previously isolated from $S$. aegyptius L. (Gharbo and Habib 1969; Habib 1981). Our present investigation of $S$. aegyptius var. discoideus resulted in identification of 4 alkaloids viz. senecionine, senecivernine, integerremine and retrorsine and no solid evidence was obtained for the presence of otosenine and riddelliine. The highest total alkaloid contents were found in flower heads (Table II). Before $\mathrm{Zn}$ reduction, the dichloromethane extracts had yielded only relatively small amount of PAs indicating that most of these alkaloids were found in the intact plants in $\mathrm{N}$-oxide forms. Thus, comparative evaluation of PA patterns of S. aegyptius L. and $S$. aegyptius var. discoideus Boiss. are clearly distinguished by their PAs contents.

As the ${ }^{13} \mathrm{C}$ NMR spectrum of $\mathbf{4}$ contained 18 resonances, apparently corresponding to 18 carbons (Table III) and its ${ }^{1} \mathrm{H}$ NMR spectrum integrated for 25 hydrogens, it was inferred that the molecular composition was $\mathrm{C}_{18} \mathrm{H}_{25} \mathrm{NO}_{5}$. MS, ${ }^{1} \mathrm{H}$ - and ${ }^{13} \mathrm{C}$ NMR spectra of compound 4 were identical
Tabele III. ${ }^{1} \mathrm{H}$ and ${ }^{13} \mathrm{C}$ NMR spectral data of senecionine (4).

\begin{tabular}{lll}
\hline Position & ${ }^{1} \mathrm{H}$ NMR & ${ }^{13} \mathrm{C}$ NMR \\
\hline 1 & - & 131.94 \\
2 & $6.151 \mathrm{H}, \mathrm{d}, J=5.1 \mathrm{~Hz}$ & 136.27 \\
3 & $\mathrm{H}-3 \alpha 3.38,1 \mathrm{H}, \mathrm{m}$ & 62.92 \\
& $\mathrm{H}-3 \beta 3.92,1 \mathrm{H}, \mathrm{br} \mathrm{d}, J=15.9 \mathrm{~Hz}$ & \\
5 & $\mathrm{H}-5 \alpha 2.501 \mathrm{H}, \mathrm{m}$ & 53.21 \\
& $\mathrm{H}-5 \beta 3.331 \mathrm{H}, \mathrm{m}$ & \\
6 & $\mathrm{H}-6 \alpha 3.251 \mathrm{H}, \mathrm{m}$ & 34.95 \\
& $\mathrm{H}-6 \beta 2.351 \mathrm{H}, \mathrm{m}$ & \\
7 & $4.991 \mathrm{H}, \mathrm{d}, J=3 \mathrm{~Hz}$ & 75.04 \\
8 & $4.271 \mathrm{H}, \mathrm{br} \mathrm{s}$ & 77.73 \\
9 & $\mathrm{H}-9 \alpha 4.01 \mathrm{br} \mathrm{d}, J=11.1 \mathrm{~Hz}$ & 60.69 \\
& $\mathrm{H}-9 \beta 5.46,1 \mathrm{H}, \mathrm{d}, J=7.2 \mathrm{~Hz}$ & 178.37 \\
11 & - & 76.85 \\
12 & - & 38.46 \\
13 & $1.65,1 \mathrm{H}, \mathrm{m}$ & 38.58 \\
14 & $\mathrm{H}-14 \alpha 1.72,1 \mathrm{H}, \mathrm{m}$ & \\
& $\mathrm{H}-14 \beta 2.14,1 \mathrm{H}, \mathrm{m}$ & 133.22 \\
15 & - & 167.68 \\
16 & - & 25.39 \\
18 & $1.283 \mathrm{H}, \mathrm{s}$ & 11.27 \\
19 & $0.873 \mathrm{H}, \mathrm{d}, J=6.3 \mathrm{~Hz}$ & 134.11 \\
20 & $5.691 \mathrm{H}, \mathrm{q}, J=6.6 \mathrm{~Hz}, 7.2 \mathrm{~Hz}$ & 15.20 \\
21 & $1.803 \mathrm{H}, \mathrm{d}, J=6.9 \mathrm{~Hz}$ & \\
& &
\end{tabular}

with those reported for senecionine (Witte et al., 1993; Logie et al., 1994; Jones et al., 1982; Roeder 1990).

\section{Acknowledgement}

Thanks are due to Prof. Dr. M. Wink (University of Heidelberg, Germany) for providing facilities for GLC and GLC-MS and Dr. H. Abdel Baset for identification of plants. 
Bicchi C., Rubiolo P., Frattini C., Sandra P. and David F. (1991), Off-line supercritical fluid extraction and capillary gas chromatography of pyrrolizidine alkaloids in Senecio species. J. Nat. Prod. 54, 941-945.

Borstel K., Witte L. and Hartmann T. (1989), Pyrrolizidine alkaloid patterns in populations of Senecio vulgaris, S. vernalis and their hybrids. Phytochemistry 28 $1635-1638$.

El-Shazly A., Sarg T., Ateya A., Abdel Aziz E., ElDahmy S., Witte L. and Wink M. (1996a), Pyrrolizidine alkaloids from Echium setosum and Echium vulgare. J. Nat. Prod. 59, 310-313.

El-Shazly A., Sarg T., Ateya A., Abdel Aziz E., ElDahmy S., Witte L. and Wink M. (1996b), Pyrrolizidine and tetrahydroisoquinoline alkaloids from Echium humile. Phytochemistry 42, 225-230.

El-Shazly A., Sarg T., Ateya A., Abdel Aziz E., Witte L. and Wink M. (1996c), Pyrrolizidine alkaloids of $C y$ noglossum officinale and Cynoglossum amabile (Family Boraginaceae). Biochem. Syst. Ecol. 24, 415-421.

El-Shazly A., Sarg T., Witte L. and Wink M. (1996d), Pyrrolizidine alkaloids from Cynoglossum creticum. Phytochemistry 42, 1217-1221.

El-Shazly A., El-Domiaty M., Witte L. and Wink M. (1998), Pyrrolizidine alkaloids in members of the Boraginaceae from Sinia (Egypt). Biochem. Syst. Ecol. 26, 619-639.

El-Shazly A., Abdel-All M., Tei A. and Wink M. (1999), Pyrrolizidine alkaloids from Echium rauwolfii and Echium horridum (Boraginaceae). Z. Naturforsch. 54 C, 295-300.

Gharbo A. S. and Habib A. M. (1969), Phytochemical investigation of Egyptian Senecio species. Part II. Alkaloids of Senecio aegyptius, S. desfontainei, S. vulgaris, $S$. petasitis and S. mikanioides. Lloydia 32, 503-508.

Habib A. M. (1974), Senecionine, seneciphylline, jacobine and otosenine from Senecio cineraria. Planta Med. 26, 279-282.

Habib A. M. (1981), Alkaloids from Senecio aegyptius and $S$. desfontainei. Planta Med. 43, 290-292.

Hartmann T. and Witte L. (1995), Chemistry, biology and chemecology of pyrrolizidine alkaloids. In: Alkaloids: Chemical and Biological Prespective (Pelletier S. W., ed.). Pergamon, Oxford, Vol. 9, pp. 155- 233.

Jones A. J., Culvenor C. C. J. and Smith W. (1982), Pyrrolizidine alkaloids- A carbon-13 N.M. R. study. Aust. J. Chem. 35, 1173-84.

Kovats E. (1958), Gas-Chromatographische Charakterisierung organischer Verbindungen. Teil I: RetentionsIndices aliphatischer Halogenide, Alkaloide, Aldehyde und Ketone. Helv. Chim. Acta 41, 1915-1932.
Krebs H. C., Carl T. and Habermehl G. (1996), Pyrrolizidine alkaloid composition in six Brazilian Senecio species. Phytochemistry 43, 1227-1229.

Logie C. G., Grue M. R. and Liddell J. R. (1994), Proton NMR spectroscopy of pyrrolizidine alkaloids. Phytochemistry 37, 43-109.

Mattocks A. R. (1986), Chemistry and Toxicology of Pyrrolizidine Alkaloids. Academic Press, London.

Pieters L. A. and Vlietinck A. J. (1988), Spartioidine and usaramine, two pyrrolizidine alkaloids from Senecio vulgaris. Planta Med. 54, 178- 179.

Ray A. C., Williams H. J. and Reagor J. C. (1987), Pyrrolizidine alkaloids from Senecio longilobus and Senecio glabellus. Phytochemistry 26, 2431-2433.

Resch J. F., Goldstein S. A. and Meinwald J. (1983), Otosenine from Senecio aureus. Planta Med. 47, 255.

Rizk A. M. (1990), Naturally Occurring Pyrrolizidine Alkaloids. CRC Press, Boca Raton, FL, USA

Roberts M. F. and Wink M. (1998), Alkaloids: Biochemistry, Ecology, and Medicinal Applications. Plenum, New York and London.

Roeder E. (1990), Carbon-13 NMR spectroscopy of pyrrolizidine alkaloids. Phytochemistry 29, 11-29.

Roeder E. (1995), Medicinal plants in Europe containing pyrrolizidine alkaloids. Pharmazie 50, 83-98.

Roeder E. (1999), Analysis of pyrrolizidine alkaloids. Curr. Org. Chem. 3, 557-576.

Roeder E. and Abdel Ghani A. (1990), Pyrrolizidine alkaloids from Petasites paradoxus (Retz.) Baumg. Sci. Pharm. 58, 403-407.

Stelljes M. E., Kelley R. B., Molyneux R. J. and Seiber J. N. (1991). GC-MS determination of pyrrolizidine alkaloids in four Senecio species. J. Nat. Prod. 54, $759-773$.

Tei A. (2000), Identifizierung und Strukturaufklärung von Alkaloiden und anderen Sekundärstoffen mit Hilfe von GC-MS und Kernresonanzspektroskopie. Dissertation, Ruprecht-Karls-Universität Heidelberg, Germany.

Witte L., Ernst L., Adam H. and Hartmann T. (1992), Chemotypes of two pyrrolizidine alkaloid-containing Senecio species. Phytochemistry 31, 559-565.

Witte L., Rubiolo P., Bicchi C. and Hartmann T. (1993), Comparative analysis of pyrrolizidine alkaloids from natural sources by gas chromatography-mass spectrometry. Phytochemistry 32, 187-196. 